\title{
Transepithelial corneal cross-linking assisted by two continuous cycles of iontophoresis for progressive keratoconus in adults: retrospective 5 -year analysis
}

\author{
Huping Wu ${ }^{1,2,3} \cdot$ Shunrong Luo ${ }^{1,3} \cdot$ Xie Fang $^{1,3} \cdot$ Xumin Shang $^{1,3} \cdot$ Zhiwen Xie $^{1} \cdot$ Xianwen Xiao $^{1,3} \cdot$ Zhirong Lin $^{1,2,3}$ (D) \\ Zuguo Liu $^{1,2}$
}

Received: 4 May 2020 / Revised: 18 July 2020 / Accepted: 23 July 2020 / Published online: 29 July 2020

(C) The Author(s) 2020

\begin{abstract}
Purpose The aim of this study is to compare the long-term effects of transepithelial corneal crosslinking with two continuous cycles of iontophoresis (EI-CXL) and conventional corneal crosslinking (C-CXL) in adults with progressive keratoconus.

Methods A retrospective analysis was conducted in adults who underwent C-CXL or EI-CXL between 2013 and 2015 . Visual acuity, corneal tomography, anterior segment optical coherence tomography, in vivo corneal confocal microscopy (IVCM), and endothelial cell count (ECC) were performed preoperatively and 5 years postoperatively.

Results Sixty-eight patients with a mean age of (24.3 \pm 3.8$)$ years were included, 34 for each group. After CXL, UCVA or BCVA remained stable, while the spherical diopter, cylinder diopter, spherical equivalent, and $K_{\max }$ significantly decreased at 1,2 , and 3 years in both groups than baseline $(P<0.05)$. No significant differences were found in any refractive or tomographic parameters as well as the minimal corneal thickness between groups during follow-up. At 5 years, $K_{\max }$ was slightly higher in EI-CXL group $(58.16 \pm 6.28)$ than that of C-CXL group (57.46 \pm 4.98$)$. At 3 and 5 years, the minimal corneal thickness in C-CXL group was still significantly lower than baseline $(P<0.05)$. IVCM demonstrated the demarcation zone at a mean depth of $(302.0 \pm 41.7)$ $\mu \mathrm{m}$ after C-CXL, and at $(251.2 \pm 28.1) \mu \mathrm{m}$ after EI-CXL $(P<0.001)$. Keratocyte repopulation was detectable at all follow-up timepoint in both groups. Postoperative complications including progression were recorded in 6 patients (11.7\%) after C-CXL and 3 patients $(8.8 \%)$ after EI-CXL. ECC remained stable in both groups.

Conclusion EI-CXL showed approximate efficacy with C-CXL in stabilizing progressive keratoconus in adults. EI-CXL has the potential to be a preferable transepithelial protocol.
\end{abstract}

Keywords Progressive keratoconus · Iontophoresis · Transepithelial corneal crosslinking · Tomography · In vivo confocal scanning microscopy

\begin{abstract}
Key message
Most previous data revealed inferior efficacy of transepithelial corneal crosslinking assisted by iontophoresis when compared to conventional corneal crosslinking. The limited riboflavin penetration into the corneal stroma is considered as one of the critical inadequacies of standard transepithelial iontophoresis.

In this retrospective five-year analysis, no significant differences of the long-term refractive and visual outcome were found between conventional corneal crosslinking and transepithelial corneal crosslinking assisted by two continuous cycles of iontophoresis. Improved stromal penetration depth was achieved by two continuous cycles of iontophoresis.
\end{abstract}

Zhirong Lin

charlie.lyn668@foxmail.com

Zuguo Liu

zuguoliu@xmu.edu.cn

1 Eye Institute and Affiliated Xiamen Eye Center of Xiamen University, Xiamen, China
2 Fujian Provincial Key Laboratory of Ophthalmology and Visual Science, Xiamen, China

3 Fujian Provincial Key Laboratory of Corneal \& Ocular Surface Diseases, Xiamen, China 


\section{Introduction}

Keratoconus (KC) has long been considered as a progressive, non-inflammatory corneal thinning and ectasia with reduced biomechanical stability, which may lead to severe visual impairment in young and even pediatric patients. In very severe cases, lamellar or penetrating corneal transplantation is the final therapeutic option to regain vision. In recent years, corneal collagen crosslinking (CXL) has been recognized as a safe and effective treatment to delay or halt further progression of $\mathrm{KC}$ and can reduce the need of keratoplasty [1]. During $\mathrm{CXL}$, riboflavin interacts with ultraviolet-A light to create crosslinking of protein fibrils followed by formation of interchain disulfide bonds, thus arresting the progression of corneal ectasia by increasing the biomechanical stability of the cornea. CXL has been considered as one of the standard treatments of progressive keratoconus worldwide.

Various protocols [1] of CXL have been extensively investigated and applicated. CXL using classic Dresden's protocol with epithelium removal (conventional CXL) showed long-term efficacy of stabilization and improvement for KC. However, postoperative complications using standard epi-off protocol [2-5], such as corneal haze, sterile corneal infiltrates, recurrent erosion syndrome, have been reported and should be taken into consideration. To reduce the risk of postoperative complications, epithelium-on(epi-on) protocols assisted by iontophoretic delivery or transepithelial riboflavin were brought into sight and were considered by some researchers to be a better choice.

Iontophoresis, in which an electrical gradient is used to drive negatively charged riboflavin molecules across the intact epithelium, may further enhance riboflavin penetration in transepithelial CXL. Laboratory and clinical studies [6-9] of iontophoresis have been encouraging, demonstrating increased transepithelial penetration of riboflavin and improvement of corneal biomechanics. Nevertheless, most studies showed inferior results of standard protocol of iontophoresis when compared to epi-off protocol [10-13]. The limited depth of riboflavin penetration and lower concentration of riboflavin in the corneal stroma were the critical inadequacies of standard iontophoresis protocol [14]. To overcome this shortcoming, transepithelial CXL assisted by two continuous cycles of iontophoresis [15] was taken into consideration, and some study showed better short-term outcome than that by standard iontophoresis [15]. Theoretically, two continuous cycles of standard iontophoresis (enhance iontophoresis) allowed time for riboflavin to penetrate and diffuse more posteriorly. However, the long-term effect of this modified iontophoretic protocol remained unclear.

This study aimed to compare the long-term efficacy of enhanced iontophoresis-assisted transepithelial corneal crosslinking (EI-CXL) and conventional corneal crosslinking (C-CXL) in adults with progressive keratoconus, as well as the characteristics in visual acuity, corneal topography, and morphological alteration.

\section{Methods}

\section{Patients and criteria}

This retrospective nonrandomized study comprised the patients who were diagnosed with progressive keratoconus and underwent a C-CXL or EI-CXL procedure between January 2013 and January 2015 at the affiliated Xiamen Eye Center of Xiamen University, China. The diagnosis of keratoconus was established in concordance with the consensus of keratoconus and ectatic corneal diseases [16]. The following features were defined to be the inclusion criteria for the study: (1) diagnosed as patients as progressive keratoconus and aged $\geq 14$ years; (2) at least 60 months of follow-up postoperatively. For patients with bilateral progressive keratoconus, only the right eye was included. Exclusion criteria were (1) patients with a minimal corneal thickness lower than $400 \mu \mathrm{m}$, (2) patients aged $<14$ years, (3) patients with maximum keratometry $\left(K_{\max }\right)$ higher than 60.0 diopter (D), and (4) patients with irregular or incomplete follow-up. Written informed consent was obtained from patients themselves. The study and surgical protocol were both approved by the hospital's ethics committee and were performed according to the tenets of the Declaration of Helsinki.

Before diagnosed as progressive keratoconus [17-20], patients were followed up for at least 12 months. Besides an increase in $K_{\max }$ of $\geq 1.0 \mathrm{D}$, progressive keratoconus was defined with any of the following criteria that occurred during the 12 months: (1) a 5\% or more reduction in thickness of the thinnest point of the cornea obtained by corneal tomography; (2) an increase in cylindrical value of $\geq 1.0 \mathrm{D}$, or in spherical equivalent $\geq 0.5 \mathrm{D}$; and (3) loss of at least 2 lines of vision in BCVA within 12 months.

\section{Surgical procedures of C-CXL and EI-CXL}

Before surgery, topical $0.1 \%$ pilocarpine eye drops were instilled $30 \mathrm{~min}$ before surgery. Topical $0.5 \%$ proparacaine hydrochloride eye drops were instilled twice before surgery (every $5 \mathrm{~min}$ ). All surgical procedures were performed in a sterile operating room.

For C-CXL, the central $9.0 \mathrm{~mm}$ (diameter) corneal epithelium was removed by mechanical debridement using a blunt spatula. After epithelial abrasion, $0.1 \%$ solution of riboflavin in $20 \%$ dextran (Ricrolin, SOOFT, Italy) was applied to the cornea every $1 \mathrm{~min}$ for $20 \mathrm{~min}$. The central cornea was then irradiated (UVX-2000, IROC, Switzerland) with a light spot of $9 \mathrm{~mm}$ diameter for $10 \mathrm{~min}$ at a $9 \mathrm{~mW} / \mathrm{cm}^{2} \mathrm{UV}$-A light $(5.4 \mathrm{~J} /$ $\mathrm{cm}^{2}$ surface dose). Riboflavin solution was further applied every 2 min during the UV-A irradiation. At the end of surgery, a soft bandage contact lens was placed for 1 week.

For EI-CXL, the return electrode was affixed to the skin of frontal region, while the corneal iontophoresis electrode was attached to the cornea by a vacuum adsorption device (SOOFT, 
Italy). The corneal electrode was filled with approximately $0.5 \mathrm{~mL}$ of $0.1 \%$ riboflavin solution (Ricrolin ${ }^{+}$, SOOFT, Italy), which was specifically designed for iontophoretic delivery of riboflavin, from the open proximal side until the stainless steel mesh was completely immersed. After that, the device was connected to a constant current generator (I-ON XL, SOOFT, Italy) set at $1 \mathrm{~mA}$ current. The total dose of $10 \mathrm{~mA} / 10 \mathrm{~min}$ (continuous two cycles of standard iontophoretic delivery) was monitored by the generator. After completion of iontophoresis, the UV light was then focus on the apex of the cornea through the four-spot alignment system. The cornea was irradiated at the same dose of UV-A as C-CXL. During irradiation, drops of balanced solution were applied to the cornea every $1 \mathrm{~min}$ to keep moisture and rinse away residual riboflavin. No soft contact lens was placed after surgery.

For both protocols, tobramycin and dexamethasone eye ointment (Alcon, Novartis, Switzerland) was applied to the conjunctival sac postoperatively. Subsequent treatment included $0.5 \%$ loteprednol and tobramycin eye drops four times per day and tapered over 4 weeks, topical artificial tears of $0.3 \%$ hyaluronate sodium four times per day for at least 8 weeks. Patients were investigated before surgery and at 1 , 2,3 , and 5 years after CXL treatment as follows.

\section{Postoperative follow-up}

In the preoperative and postoperative examinations, the following parameters were accessed: uncorrected distance visual acuity (UCVA), best corrected distance visual acuity (BCVA), slit-lamp microscopy examination including corneal fluorescein sodium staining (BQ900IM9900, Haag-Streit, Switzerland), corneal tomography and pachymetry (Pentacam HR 70900, Oculus, Wetzlar, Germany), anterior segment optical coherence tomography (AS-OCT, Visante OCT, Carl Zeiss Meditec Inc., Germany), endothelial biomicroscope (SP-3000P, Topcon, Tokyo, Japan), and in vivo corneal confocal microscopy (IVCM, HRT3/Rostock Cornea Module, Heidelberg Engineering $\mathrm{GmbH}$, Germany). $K$ values ( $K_{\max }, \mathrm{K} 1$ and $\mathrm{K} 2$ ) and minimum pachymetry values were derived from the tomography data. All patients were assessed at baseline and followed up for 5 years postoperatively. The UCVA and BCVA were converted to logarithm of the minimum angle of resolution ( $\log$ MAR) units for statistical analysis.

\section{Statistical analysis}

The data was imported to the Statistical Package for Social Sciences (SPSS Inc., Chicago, IL, version 16.0) for analysis. Two-way repeated measures ANOVA and student's $t$ test were used for statistical comparisons as appropriate. Bonferroni correction was made for multiple comparisons. For binary outcomes, the stratified Cochran chi-square test was used for comparisons of proportions between groups. The significance level was set at $<0.05$.

\section{Results}

\section{Demographics of patients}

A total of 133 patients with keratoconus underwent C-CXL or EI-CXL in the affiliated Xiamen Eye Center of Xiamen University between January 2013 and January 2015. In these patients, 65 treated cases were excluded in the study by reasons of age less than 14 years, minimal corneal thickness lower than $400 \mu \mathrm{m}, K_{\max }$ higher than $60.0 \mathrm{D}$, and rare or irregular followup. Eventually, a total of 68 patients ( 68 eyes) were included, 34 patients for each group. Patient demographics are listed in Table 1. The baseline values of the two groups were comparable, including age, sex ratio, ratio of right eyes, and ratio of history with allergic conjunctivitis (all $P>0.05$ ).

\section{Refractive and tomographic changes after C-CXL and EI-CXL}

Comparative analysis of the UCVA and BCVA as well as refractive parameters at all follow-up period was shown in Table 2 . After CXL, UCVA or BCVA remained stable in each group, and no statistical difference was found between the two groups. After CXL, the spherical, cylinder diopter as well as the spherical equivalent value significantly decreased at 1,2 , and 3 years in both group when compared with baseline $(P<0.05)$. Although the refractive data of spherical, cylinder diopter, and spherical equivalent showed slightly higher absolute value in the EICXL group at 5 years, no significant difference was found between the two groups at any follow-up time.
Table 1 Patients demographics for all subjects included in this study

\begin{tabular}{lllll}
\hline & C-CXL group $(n=34)$ & EI-CXL group $(n=34)$ & All patients, $(n=68)$ & $P$ value \\
\hline Age (years) & $24.9 \pm 4.0$ & $23.8 \pm 3.6$ & $24.3 \pm 3.8$ & 0.236 \\
Sex ratio (M/F) & $20 / 14$ & $16 / 18$ & $36 / 30$ & 0.331 \\
Side (OD/OS) & $15 / 19$ & $16 / 18$ & $31 / 37$ & 0.808 \\
$K_{\max }$ & $58.37 \pm 4.96$ & $58.26 \pm 5.78$ & $58.31 \pm 5.34$ & 0.917 \\
AC (Y/N) & $25 / 9$ & $23 / 11$ & $48 / 20$ & 0.595 \\
\hline
\end{tabular}

$M / F$ male/female, $A C$ allergic conjunctivitis; data was presented with mean \pm standard deviation 


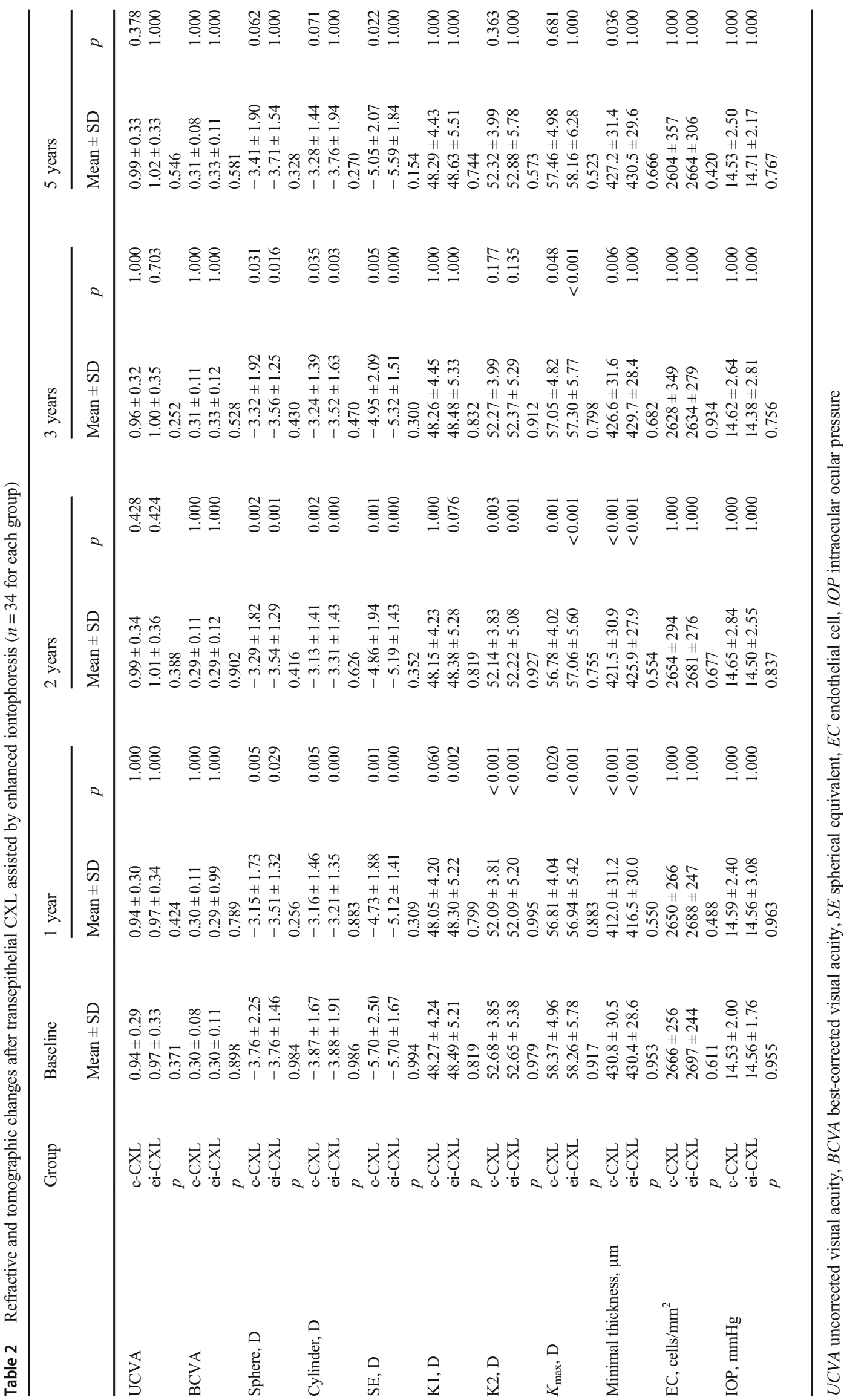


After CXL, corneal flattening was seen with significant decreases in $K_{\max }$ (at 1,2 , and 3 years, $P<0.05$ ) and $\mathrm{K} 2$ (at 1 and 2 years, $P<0.01$ ) in each group when compared with the baseline; however, the $K_{\max }$ and $\mathrm{K} 2$ values at 5 years after surgery were comparable to baseline $(P>0.05)$. No significant difference in $\mathrm{K} 1, \mathrm{~K} 2$, or $K_{\max }$ was found between the two groups at any follow-up time $(P>0.05)$. The corneal thickness of the thinnest point significantly reduced at all the follow-up time after C-CXL $(P<0.05)$, but reduced only at 1 and 2 years after EI-CXL $(P<0.001)$. Likewise, no difference was found in corneal thickness of the thinnest point between the two groups at any follow-up time $(P>0.05)$.

\section{Structural alteration in the corneal stroma}

At 1 month after CXL, demarcation line was visible in a low proportion of patients treated by EI-CXL $(6 / 34,17.6 \%)$ at a mean depth of about $230 \mu \mathrm{m}$, whereas it was visible in most of the patients $(25 / 34,73.5 \%)$ at a mean depth of about $300 \mu \mathrm{m}$ by C-CXL. IVCM images also demonstrated the demarcation zone between the treated and untreated corneal stroma, at a mean depth of $(302.0 \pm 41.7) \mu \mathrm{m}$ after C-CXL and of $(251.2$ $\pm 28.1) \mu \mathrm{m}$ after EI-CXL. The depth of corneal demarcation zone detected by IVCM was significantly deeper after C-CXL than after EI-CXL $(P<0.001)$. Representative IVCM images at 3 years after C-CXL and EI-CXL were shown (Fig. 1).

a

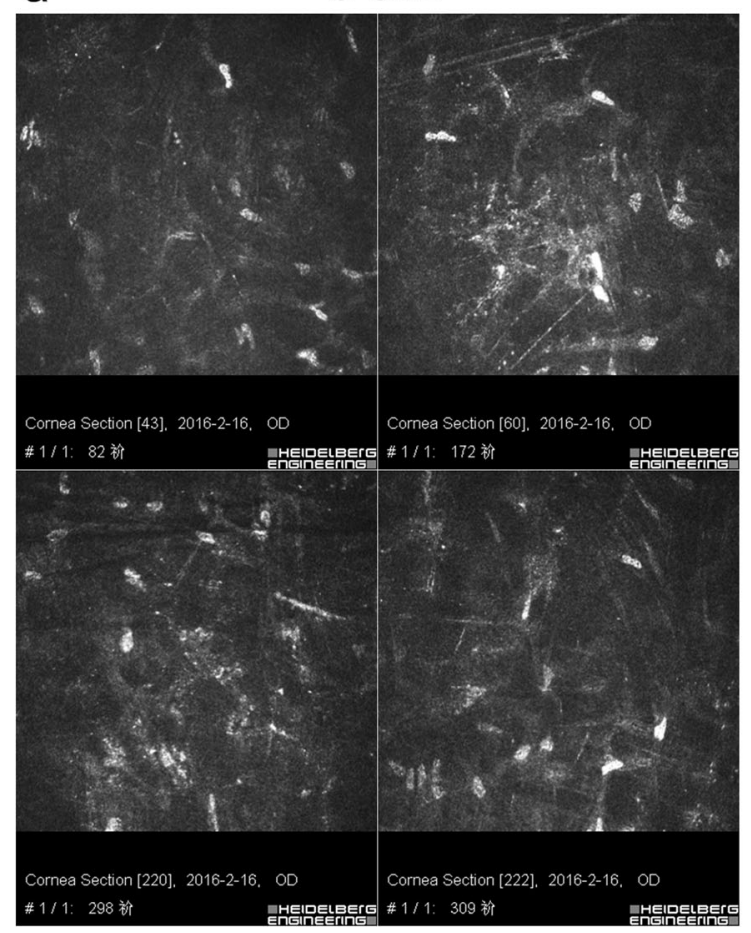

Fig. 1 In vivo confocal microscopy scans of the corneal stroma at 3 years after C-CXL (patient a) and EI-CXL (patient b). In patient a, consecutive scans at different corneal depth: 82, 172, 298, and $309 \mu \mathrm{m}$. In patient b,
Representative IVCM images at $200 \mu \mathrm{m}$ measured from epithelial surface of each follow-up time were also shown in Fig. 2. Keratocyte population was detectable but decreased at 1, 2, 3, and 5 years after C-CXL (Fig. 2b-f) and EI-CXL (Fig. $2 g-j$ ) when compared to the baseline (Fig. 2 a and f). Activated keratocytes with elongated membrane processes and surrounded hyperreflective collagen fibers were detectable in the first postoperative year for both protocols. At 2, 3 , and 5 years after surgery, cell repopulation with hyperreflective needle-shaped micro-bands or micro-striate reflections could be observed for both protocols, but the cell repopulation appeared to be more apparent in EI-CXL group at 5 years (Fig. 2j) than that in C-CXL group (Fig. 2e).

\section{Postoperative complications}

After C-CXL, all patients reported obvious pain and discomfort in the first week after surgery, while good toleration to EI-CXL surgery was recorded in 31 patients $(31 / 34,91.2 \%)$. Central corneal epithelial exfoliation ( $9 \mathrm{~mm}$ diameter) after EI-CXL was found in 1 patient $(2.9 \%)$ with a history of allergic conjunctivitis on day 1 after surgery, but gradually recovered after 7 days. Sterile corneal infiltrate $(1 / 34,2.9 \%)$ and temporary corneal haze $(3 / 34,8.8 \%)$ was recorded after C-CXL, but none ( $0 \%)$ after EICXL. No persistent corneal haze, recurrent epithelium erosion, corneal melting, or perforation were noted during the period of follow-up after both CXL protocols. Endothelial cell density and

b

EI-CXL

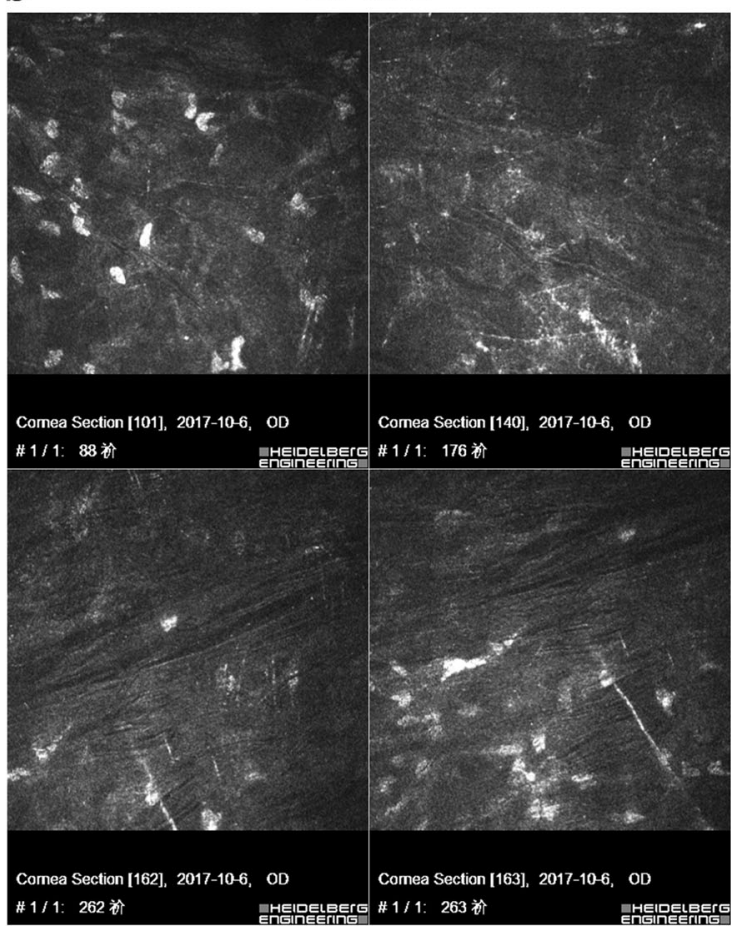

consecutive scans at similar corneal depth: $88,176,262$, and $263 \mu \mathrm{m}$. The transition zone from acellular to cellular corneal stroma was at 298 and $262 \mu \mathrm{m}$ in patient a and $\mathrm{b}$, respectively 


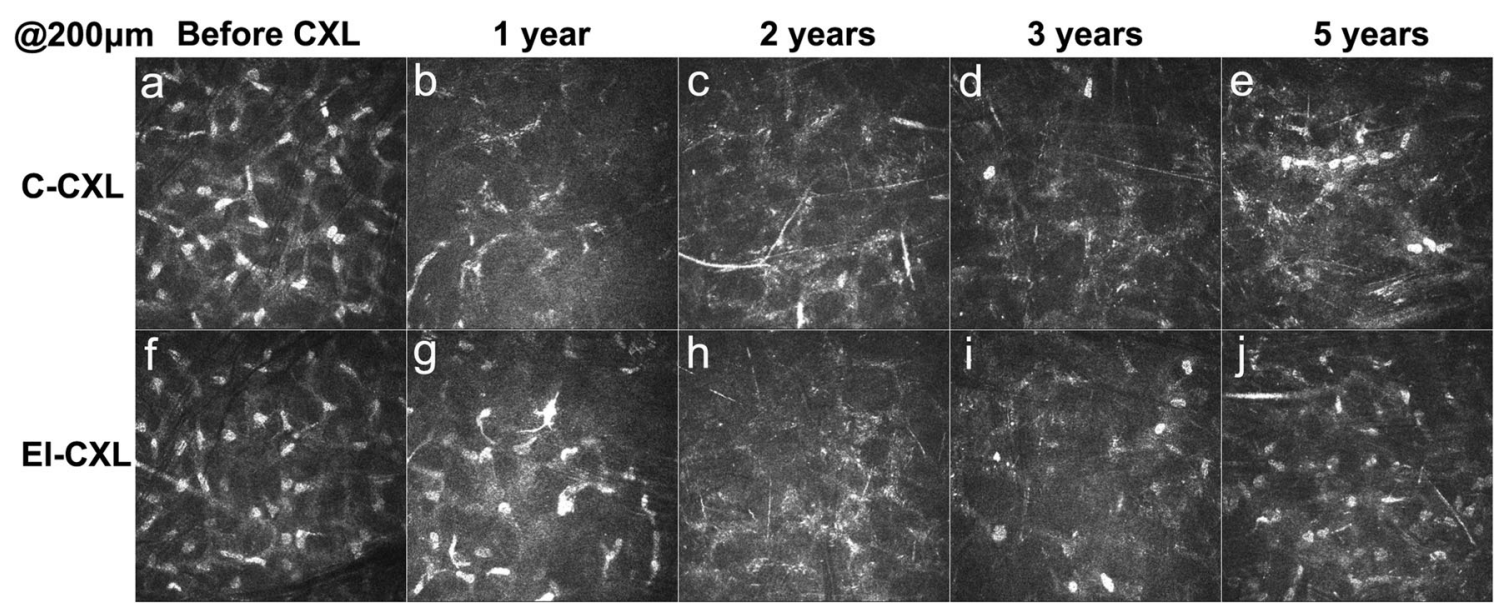

Fig. 2 Representative images of IVCM at the depth of $200 \mu \mathrm{m}$ for CCXL $(\mathbf{a}-\mathbf{e})$ and EI-CXL $(\mathbf{f}-\mathbf{j})$. Keratocyte repopulation was detectable at all follow-up timepoint after C-CXL and EI-CXL, but the cell density was lower than baseline. Hyperreflective needle-shaped reflection could be

intraocular pressure remained stable after both protocols. Further keratoconus progression was recorded in 2 patients after $\mathrm{C}-\mathrm{CXL}$ ( 1 after 3 years and 1 after 4 years) and 3 patients after EI-CXL ( 2 after 2 years and 1 after 3 years).

\section{Discussion}

In the past decade, although some study demonstrated that iontophoresis-assisted CXL could achieve the same clinical result as standard CXL [21], most evidences have demonstrated inferior results of standard protocol of transepithelial CXL when compared to the conventional epi-off protocol. However, our data indicated that EI-CXL achieved by doubling the iontophoresis cycle displayed approximate long-term(5 years) efficacy with C-CXL in halting the progression of keratoconus in adults. This modified transepithelial protocol might be a good alternative in the management of progressive keratoconus. To our knowledge, our study was the first to demonstrate the long-term effects of transepithelial CXL assisted by iontophoresis for $10 \mathrm{~min}$.

Riboflavin is water soluble and negatively charged at physiological $\mathrm{pH}$. In transepithelial CXL, iontophoresis can be applied effectively to enhance riboflavin penetration. Existing recommendations for iontophoresis in transepithelial CXL utilize $1 \mathrm{~mA}$ for $5 \mathrm{~min}$ with a $0.1 \%$ riboflavin solution. Standard iontophoresis allowed riboflavin imbibition with one-half the concentrations of the C-CXL technique in a rabbit model [8]. Variables in protocols of iontophoresis are able to obtain improved riboflavin penetration. For example, the use of the cationic surfactant benzalkonium chloride has been shown with percutaneous iontophoresis to have a synergistic effect on the transport of anions [6]. An ex vivo study utilized two cycles of iontophoresis each followed by a 5-min soak period to allow time for riboflavin to diffuse more posteriorly, and the stromal riboflavin concentrations were found to be similar to epithelium-off controls [22]. observed for both protocols at 2 and 3 years after surgery. At 5 years after surgery, cell repopulation appeared more apparent in EI-CXL group (j) than that in C-CXL group (e)

Transepithelial CXL assisted by two continuous cycles of iontophoresis also showed better short-term outcome than that by standard iontophoresis [15]. In our study, no significant differences of the long-term refractive and visual outcome were found between C-CXL and EI-CXL group. The cessation of keratoconus progression with up to 5 years follow-up demonstrated the potential of this modified iontophoretic protocol.

The depth of the acellular zone of anterior corneal stroma after CXL has been correlated with the effectiveness of the CXL treatment [23-25]. The limited penetration depth of riboflavin is one of the critical inadequacies of transepithelial CXL with standard iontophoresis protocol. The penetration depth ranged from 100 to $240 \mu \mathrm{m}$ in most clinical studies [25-29]. In our previous study [30], the penetration depth of transepithelial CXL using the same iontophoretic device for $5 \mathrm{~min}$ was about $133 \mu \mathrm{m}$ from the corneal surface. The penetration depths in these iontophoretic protocols were all shallower when compared to that in conventional CXL. The huge range of different penetration depth among these reports might be contributed to the variety of iontophoretic devices, riboflavin concentration, and UV radiation parameters used in the CXL surgery. In a series of laboratory investigation, it has been shown that by increasing riboflavin concentration, iontophoresis application times, and allowing short periods of time for riboflavin, which in initially deposited only into the epithelium and anterior stroma, to diffuse deeper into the stroma, concentrations of up to $80 \%$ of that achieved with epithelium-off application with a homogeneous distribution throughout the stroma can be achieved [26]. Apparently increased penetration depth could be achieved by repeated iontophoresis. In this study, IVCM showed an average penetration depth of $251 \mu \mathrm{m}$ in EI-CXL, which achieved an increase of almost $90 \%$ of that achieved with iontophoresis using riboflavin $0.1 \%$ and $0.1 \mathrm{~mA}$ for $5 \mathrm{~min}$ in our previous standard iontophoresis $(133 \mu \mathrm{m})$. The penetration depth in EI-CXL was also far deeper than those achieved with any of the transepithelial 
CXL protocols using chemical enhancers. With such improved transepithelial penetration depth, EI-CXL achieved approximate results to $\mathrm{C}-\mathrm{CXL}$ during the follow-up period of 5 years. However, it should be noted that the penetration depth was still more superficial in EI-CXL group than that of C-CXL group.

The statistical analysis showed no differences in the tomographic and refractive parameters between group during the 5year follow-up (Table 2). Although improvement of $K_{\max }, \mathrm{K} 2$, and SE were observed after surgery within both groups, no significant improvement of BCVA was observed in either group of this study. In addition, the refractive data of spherical, cylinder diopter, spherical equivalent, and $K_{\max }$ showed higher absolute value in the EI-CXL group at 5 years, even though no statistical difference was found. Furthermore, there was a slight increase of corneal stromal cell density and loss of hyperreflective needle-shaped reflection at 5 years after EICXL (Fig. 2e), while that in C-CXL group remained almost unchanged (Fig. 2j). This subtle difference of representative IVCM images noted at 5 years after surgery implied that the corneal collagen turnover might happen earlier in EI-CXL group. The earlier turnover of corneal collagen, the more superficial demarcation line in combination with tapering refractive improvement over time after EI-CXL might indicate an inferior efficacy of the EI-CXL protocol when compared with the C-CXL protocol. Further follow-up is needed to determine whether the corneal collagen turnover may induce loss of EICXL effect duration with new corneal instability or keratoconus progression in 5 to 10 years after EI-CXL.

The main limitations of our study include the retrospective study design, lack of randomization, and a relatively small sample size that may reduce the power to further interpretation. As a regional eye center in China, our hospital treated a large number of keratoconus patients who lived far away from our hospital. Most of these patients could not guarantee the regular follow-up, increasing the difficulty to conduct a prospective study. In addition, other factors such as age < 14 years, too thin cornea, and $K_{\max }>60.0 \mathrm{D}$ further cut down the final number of included cases. Furthermore, the treatment protocol was selected and decided by individual patients, depending on their self-estimated tolerance to postoperative pain and acceptance to potential weak effect of epi-on CXL. Besides, patients with more severe keratoconus might prefer $\mathrm{C}-\mathrm{CXL}$ in order to gain possible better outcome. The lack of randomization inevitably led to selection bias.

In conclusion, transepithelial CXL assisted by enhanced iontophoresis achieved improved stromal penetration depth. Although less than that achieved by conventional epitheliumoff protocol, its considerable stromal riboflavin penetration as well as long-term tomographic and refractive improvement, without obvious postoperative complications, demonstrated the potential of EI-CXL to be a better transepithelial protocol. Randomized multicenter clinical trials are needed to further determine the long-term efficacy level.
Acknowledgements This research was supported by grants from the National Natural Science Foundation of China, Xiamen Science and Technology Program for Public Wellbeing, Xiamen Medical and Health Project, Huaxia Translational Medicine Fund for Young Scholars, and Research Project of Health and Family Planning for Youth in Fujian Province.

Availability of data and materials The datasets generated and/or analyzed during the present study are not publicly available (obtained from the affiliated Xiamen Eye Center of Xiamen University, Xiamen repository), but are available from the corresponding author upon reasonable request.

Code availability Not applicable.

Authors' contributions HW, ZRL, and ZGL conceived the study. HW, SL, XF, ND, XS, ZX, and XX collected the data. HW and ZRL did the statistical analysis of the data. SL and XF further analyzed the data. HW and SL prepared the manuscript. All the authors contributed to the manuscript and approved the final draft for submission.

Funding This research was supported by grants from the National Natural Science Foundation of China (81570816, 81570815), Xiamen Science and Technology Program for Public Wellbeing (3502Z20174003), Xiamen Medical and Health Project (3502Z20189024), Huaxia Translational Medicine Fund for Young Scholars (2018-A-006), Research Project of Health, and Family Planning for Youth in Fujian Province (2017-2-117). The funding sources had no role in the design and conduct of the study; collection, analysis and interpretation of the data; preparation, review, approv$\mathrm{al}$, and submission of the manuscript.

\section{Compliance with ethical standards}

Competing interests The authors declare that they have no competing interests.

Ethical approval This study and surgical protocol were both approved by the ethics committee of affiliated Xiamen Eye Center of Xiamen University. All procedures adhered to the Declaration of Helsinki and were conducted in accordance with the approved protocol.

Consent to participate Written informed consent was obtained from patient before his/her participation.

Consent for publication Not applicable.

Open Access This article is licensed under a Creative Commons Attribution 4.0 International License, which permits use, sharing, adaptation, distribution and reproduction in any medium or format, as long as you give appropriate credit to the original author(s) and the source, provide a link to the Creative Commons licence, and indicate if changes were made. The images or other third party material in this article are included in the article's Creative Commons licence, unless indicated otherwise in a credit line to the material. If material is not included in the article's Creative Commons licence and your intended use is not permitted by statutory regulation or exceeds the permitted use, you will need to obtain permission directly from the copyright holder. To view a copy of this licence, visit http://creativecommons.org/licenses/by/4.0/.

\section{References}

1. O'Brart DPS (2017) Corneal collagen crosslinking for corneal ectasias: a review. Eur J Ophthalmol 27:253-269. https://doi.org/ 10.5301/ejo.5000916 
2. Dhawan S, Rao K, Natrajan S (2011) Complications of corneal collagen cross-linking. J Ophthalmol 2011:869015. https://doi. org/10.1155/2011/869015

3. Evangelista CB, Hatch KM (2018) Corneal collagen cross-linking complications. Semin Ophthalmol 33:29-35. https://doi.org/10. 1080/08820538.2017.1353809

4. Sharma A, Nottage JM, Mirchia K, Sharma R, Mohan K, Nirankari VS (2012) Persistent corneal edema after collagen cross-linking for keratoconus. Am J Ophthalmol 154(922-926):e921. https://doi. org/10.1016/j.ajo.2012.06.005

5. Lam FC, Geourgoudis P, Nanavaty MA, Khan S, Lake D (2014) Sterile keratitis after combined riboflavin-UVA corneal collagen cross-linking for keratoconus. Eye (Lond) 28:1297-1303. https:// doi.org/10.1038/eye.2014.173

6. Lombardo M, Serrao S, Rosati M, Ducoli P, Lombardo G (2014) Biomechanical changes in the human cornea after transepithelial corneal crosslinking using iontophoresis. J Cataract Refract Surg 40:1706-1715. https://doi.org/10.1016/j.jcrs.2014.04.024

7. Gore DM, O'Brart D, French P, Dunsby C, Allan BD (2015) Transepithelial riboflavin absorption in an ex vivo rabbit corneal model. Invest Ophthalmol Vis Sci 56:5006-5011. https://doi.org/ 10.1167/iovs. $15-16903$

8. Cassagne M, Laurent C, Rodrigues M, Galinier A, Spoerl E, Galiacy SD, Soler V, Fournie P, Malecaze F (2016) Iontophoresis transcorneal delivery technique for transepithelial corneal collagen crosslinking with riboflavin in a rabbit model. Invest Ophthalmol Vis Sci 57:594-603. https://doi.org/10.1167/iovs.13-12595

9. Buzzonetti L, Petrocelli G, Valente P, Iarossi G, Ardia R, Petroni S (2015) Iontophoretic transepithelial corneal cross-linking to halt keratoconus in pediatric cases: 15-month follow-up. Cornea 34: 512-515. https://doi.org/10.1097/ico.0000000000000410

10. Bouheraoua N, Jouve L, El Sanharawi M, Sandali O, Temstet C, Loriaut P, Basli E, Borderie V, Laroche L (2014) Optical coherence tomography and confocal microscopy following three different protocols of corneal collagen-crosslinking in keratoconus. Invest Ophthalmol Vis Sci 55:7601-7609. https://doi.org/10.1167/iovs. 14-15662

11. Buzzonetti L, Petrocelli G, Valente P, Iarossi G, Ardia R, Petroni S, Parrilla R (2019) Iontophoretic Transepithelial collagen crosslinking versus epithelium-off collagen cross-linking in pediatric patients: 3-year follow-up. Cornea 38:859-863. https://doi.org/10. 1097/ico.0000000000001965

12. Wen DZ, Song BH, Li Q, Tu RX, Huang YY, Wang QM, McAlinden C, O'Brart D, Huang JH (2018) Comparison of epithelium-off versus transepithelial corneal collagen crosslinking for keratoconus: a systematic review and meta-analysis. Cornea 37:1018-1024. https://doi.org/10.1097/ico. 0000000000001632

13. Cerman E, Toker E, Ozarslan Ozcan D (2015) Transepithelial versus epithelium-off crosslinking in adults with progressive keratoconus. J Cataract Refract Surg 41:1416-1425. https://doi. org/10.1016/j.jcrs.2014.10.041

14. Jia HZ, Peng XJ (2018) Efficacy of iontophoresis-assisted epithelium-on corneal cross-linking for keratoconus. Int J Ophthalmol 11: 687-694. https://doi.org/10.18240/ijo.2018.04.25

15. Liao K, Hu M, Chen F, Li P, Song P, Zeng QY (2019) Clinical and microstructural changes with different iontophoresis-assisted corneal cross-linking methods for keratoconus. Int J Ophthalmol 12:219 225. https://doi.org/10.18240/ijo.2019.02.06

16. Gomes JA, Tan D, Rapuano CJ, Belin MW, Ambrosio R Jr, Guell JL, Malecaze F, Nishida K, Sangwan VS (2015) Global consensus on keratoconus and ectatic diseases. Cornea 34:359-369. https:// doi.org/10.1097/ico.0000000000000408

17. Vinciguerra R, Romano MR, Camesasca FI, Azzolini C, Trazza S, Morenghi E, Vinciguerra P (2013) Corneal cross-linking as a treatment for keratoconus four-year morphologic and clinical outcomes with respect to patient age. Ophthalmology 120:908-916. https:// doi.org/10.1016/j.ophtha.2012.10.023

18. Bikbova G, Bikbov M (2016) Standard corneal collagen crosslinking versus transepithelial iontophoresis-assisted corneal crosslinking, 24 months follow-up: randomized control trial. Acta Ophthalmol 94:e600-e606. https://doi.org/10.1111/aos.13032

19. Vinciguerra R, Romano V, Arbabi EM, Brunner M, Willoughby CE, Batterbury M, Kaye SB (2017) In vivo early corneal biomechanical changes after corneal cross-linking in patients with progressive keratoconus. J Refrac Surg 33:840-+. https://doi.org/10. 3928/1081597x-20170922-02

20. Ozer MD, Batur M, Mesen S, Tekin S, Seven E (2019)Long-term results of accelerated corneal cross-linking in adolescent patients with keratoconus. Cornea 38:992-997. https://doi.org/10.1097/ ico.0000000000001975

21. Lombardo M, Giannini D, Lombardo G, Serrao S (2017) Randomized controlled trial comparing transepithelial corneal cross-linking using iontophoresis with the Dresden protocol in progressive keratoconus. Ophthalmology 124:804-812. https://doi. org/10.1016/j.ophtha.2017.01.040

22. Gore DM, O'Brart DP, French P, Dunsby C, Allan BD (2015) A comparison of different corneal Iontophoresis protocols for promoting transepithelial riboflavin penetration. Invest Ophthalmol Vis Sci 56:7908-7914. https://doi.org/10.1167/iovs.15-17569

23. Mazzotta C, Balestrazzi A, Traversi C, Baiocchi S, Caporossi T, Tommasi C, Caporossi A (2007) Treatment of progressive keratoconus by riboflavin-UVA-inducedcross-linking of corneal collagen: ultrastructural analysis by Heidelberg retinal tomograph II in vivo confocal microscopy in humans. Cornea 26:390-397. https://doi.org/10.1097/ICO.0b013e318030df5a

24. Kymionis GD, Grentzelos MA, Plaka AD, Tsoulnaras KI, Diakonis VF, Liakopoulos DA, Kankariya VP, Pallikaris AI (2014) Correlation of the corneal collagen cross-linking demarcation line using confocal microscopy and anterior segment optical coherence tomography in keratoconic patients. Am J Ophthalmol 157:110 115. https://doi.org/10.1016/j.ajo.2013.09.010

25. Mazzotta C, Hafezi F, Kymionis G, Caragiuli S, Jacob S, Traversi C, Barabino S, Randleman JB (2015) In vivo confocal microscopy after corneal collagen crosslinking. Ocul Surf 13:298-314. https:// doi.org/10.1016/j.jtos.2015.04.007

26. Hayes S, Morgan SR, O'Brart DP, O'Brart N, Meek KM (2016) A study of stromal riboflavin absorption in ex vivo porcine corneas using new and existing delivery protocols for corneal cross-linking. Acta Ophthalmol 94:e109-e117. https://doi.org/10.1111/aos.12884

27. Bonnel S, Berguiga M, De Rivoyre B, Bedubourg G, Sendon D, Froussart-Maille F, Rigal-Sastourne JC (2015) Demarcation line evaluation of iontophoresis-assisted transepithelial corneal collagen cross-linking for keratoconus. J Refract Surg 31:36-40. https://doi. org/10.3928/1081597x-20141218-04

28. Bikbova G, Bikbov M (2014) Transepithelial corneal collagen cross-linking by iontophoresis of riboflavin. Acta Ophthalmol 92: e30-e34. https://doi.org/10.1111/aos.12235

29. Jouve L, Borderie V, Sandali O, Temstet C, Basli E, Laroche L, Bouheraoua N (2017) Conventional and Iontophoresis corneal cross-linking for keratoconus: efficacy and assessment by optical coherence tomography and confocal microscopy. Cornea 36:153162. https://doi.org/10.1097/ico.0000000000001062

30. Lin Z, Wu H, Luo S, Liu Z, Dong N, Shang X, Li X (2015) Transepithelial iontophoresis corneal collagen cross-linking for progressive keratoconus: one year results. Chinese Journal of Ophthalmology 51:677-682

Publisher's note Springer Nature remains neutral with regard to jurisdictional claims in published maps and institutional affiliations. 